\title{
Fiber Bragg Grating Sensors Integrated in Polymeric Foils
}

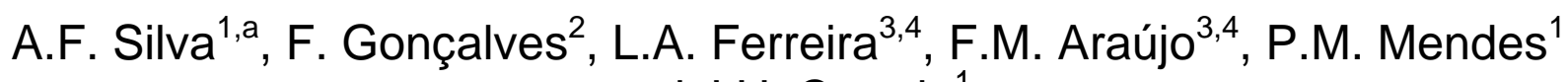 \\ and J.H. Correia ${ }^{1}$ \\ ${ }^{1}$ University of Minho, Dep. of Industrial Electronics, Campus de Azurem, Guimaraes, Portugal \\ ${ }^{2}$ TMG Automotive, Campelos, Guimaraes, Portugal \\ ${ }^{3}$ FiberSensing, Maia, Portugal \\ ${ }^{4}$ INESC Porto, Faculty of Science, University of Porto, Porto, Portugal \\ aasilva@dei.uminho.pt
}

Keywords: Optical fiber sensors, Sensors integration, Fiber bragg gratings, Structural health monitoring.

\begin{abstract}
Optical sensors have hit their maturity and a new kind of systems is being developed. This paper deals with the development of a new sensing structure based on polymeric foils and optic fiber sensors, namely the Fiber Bragg Grating sensors. Sensor integration in polymeric foils, using industrial process is the proposed goal. To achieve this goal, Finite Element Analysis was used for prototype modeling and simulation. The model was subjected to loads and restraints in order to retrieve information about stress distribution and displacement of specific points. From the simulation was possible to predict the sections where the sensor should be positioned. A prototype was then fabricated using industrial processes. Tests indicate that the polymeric foil influence on the sensor performance may exist. However, the prototype was able of transferring the full deformation to the optical sensor. Moreover, the optical sensor, which is incorporated in the polymeric foil, is fully functional with high sensitivity, 0.6 picometer by microstrain, allowing deformation measurements, up to 1.2 millimeter.
\end{abstract}

\section{Introduction}

Optical sensing technologies have associated advantages that make them very attractive in a broad range of applications. Optical fiber sensors, in particular, provide low cost solutions, with immunity to electromagnetic interference, multiplexing capabilities and a high degree of miniaturization/integration. Presently, optical fiber sensors offer an high performance alternative, in comparison to standard technologies, in many different areas, either for measuring physical parameters like strain, temperature or pressure, or for performing highly sensitive biochemical analysis [1-2]. Integrated optics devices, on the other hand, are now emerging as next generation sensing chips where virtually any parameter can be determined with high accuracy in an highly miniaturized optoelectronic device [3].

Many industries as the civil, automotive and aeronautic, among others, are already benefiting from the potential of optical sensing technologies while looking for ways to gather information from their systems status. The number and sophistication of optoelectronic systems found in smart structures is increasing at an unprecedented level. In this context, the development of advanced optoelectronic systems, with integrated sensing and data transport capabilities, is a key step for the implementation of a new generation of intelligent structures. In addition, a variety of optical sensors has been proposed for other different applications. Optical fiber sensors offer competitive and sometimes unique solutions to many different problems.

Linking textiles or textiles-polymer-laminates (artificial leather) with optical devices and electronics is more realistic than ever. An emerging new field of research that combines the strengths and capabilities of electronics, optics and polymer composites is opening new opportunities. Lower production cost, wider exploitation of integrated circuit technology and wider 
applicability to sensor arrays ensure the integration of microsensors in almost any structure, providing the desired system data.

\section{Fiber Bragg Grating}

Fiber Bragg Gratings (FBG) are periodic changes in the refraction index of the fiber core made by adequately exposing the fiber to intense UV light. The gratings produced typically have lengths of the order of $10 \mathrm{~mm}$ [4]. When an optical beam is injected into the fiber containing the grating, the wavelength spectrum corresponding to the grating pitch will be reflected, while the remaining wavelengths will pass through the grating undisturbed, as exemplified in Fig. 1 [5-6]. Since the grating period structure is sensitive to strain and temperature, these two parameters are measured by the analysis of the reflected light spectrum. This is typically done using a tunable laser containing a wavelength filter (such as a Fabry-Perot cavity) or a spectrometer [4].

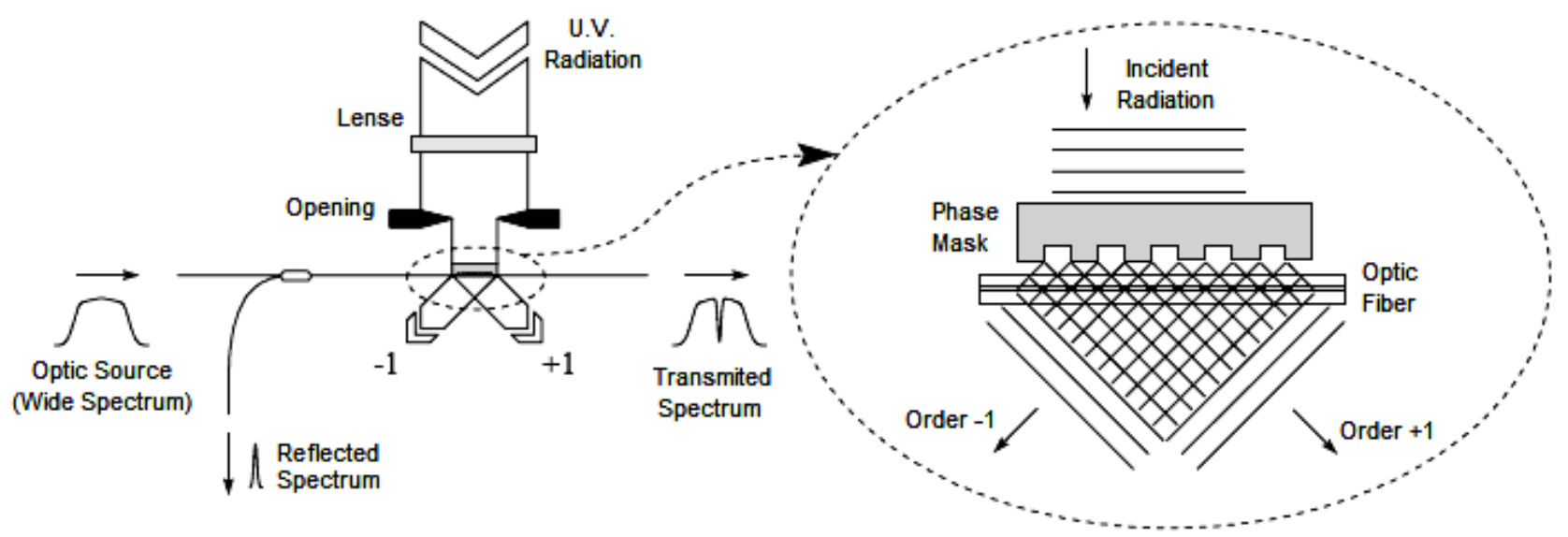

Fig. 1. Illustration of a Bragg sensor principle.

A resolution in the range of $1 \mu \varepsilon$ (micro-strain) and $0.1^{\circ} \mathrm{C}$ can be achieved with the best demodulators [4]. Since we are dealing with optical sensors that are sensitive to temperature and, in this case, also to strain by the same manner, a few issues may appear when measuring both parameters simultaneously. In this case, it is necessary to use a strain free reference grating that measures the temperature alone, in order to compensate the temperature error from the sensor network and measure the correct strain values.

A main advantage to use Bragg gratings is their multiplexing potential [5]. Many gratings can be written in the same fiber at different locations and tuned to interfere at different wavelengths. This leads to the possibility for measuring strain at different locations along a single fiber. However, since the gratings have to share the spectrum of the light, there is a trade-off between the number of gratings and the dynamic range of the measurements on each of them.

\section{Polymeric Foil}

Vinyl mixtures, or so-called Plastisols, are liquid dispersions of a finely divided thermoplastic, polyvinyl chloride resin, in a plasticizer. It is a $100 \%$ non-volatile paste-like composition and consists of a physical mixture of finely sized PVC polymer particles and liquid plasticizers, such as phthalates and epoxy oils [7-8]. These resulting pastes are highly viscous mixtures that, after heated above the curing-temperature $\left(130\right.$ to $\left.400{ }^{\circ} \mathrm{C}\right)$, become homogenized and a solid phase results. When cooled, the Plastisol provides a tough material with specific physical characteristics.

The Plastisol material is formulated to be a dense solid and elastic polymer, which retains toughness even at low temperatures. Plastisols can be formulated with hardness ranging from 30 to 90 Shore A (relative hardness of elastic materials) and tensile strength ranging from 750 to 3000 Psi 
[7-8]. It can be formulated to resist chemical attack, is self-extinguishing due to the chlorine groups, provides good weatherability and can be used as support structure when looking for flexibility

\section{Finite Element Sensing Device Model}

Finite element analysis has been carried out using specific software. A three-dimensional model of the optical fiber integrated in a polymeric foil was created using solid elements. The analyzed model had a length of $100 \mathrm{~mm}$, a width of $50 \mathrm{~mm}$ and a height of $550 \mu \mathrm{m}$. The optic fiber is $250 \mu \mathrm{m}$ thick, with a $9 \mu \mathrm{m}$ core and a $125 \mu \mathrm{m}$ cladding, and was placed in the center of the foil.

The material properties used for simulations were based on the available commercial materials properties from CES EduPack software. PVC-elastomer (shore 75A) was chosen for the polymeric foils, silica for the fiber core and cladding, and acrylate for the fiber buffer.

As the full model has a small number of elements, it was possible to simulate and observe the detailed behavior of the model along the load application time. For this reason, a 3D mesh was chosen for the simulation. The mesh was composed by 6215 brick elements that fill the model volume. The elements are defined by 20 nodes, having three degrees of freedom at each node: translations in the nodal $\mathrm{x}-, \mathrm{y}$ - and z-directions. In some sections of the model, like the fiber volume and model borders, the mesh was refined, presenting smaller elements, ensuring better results.

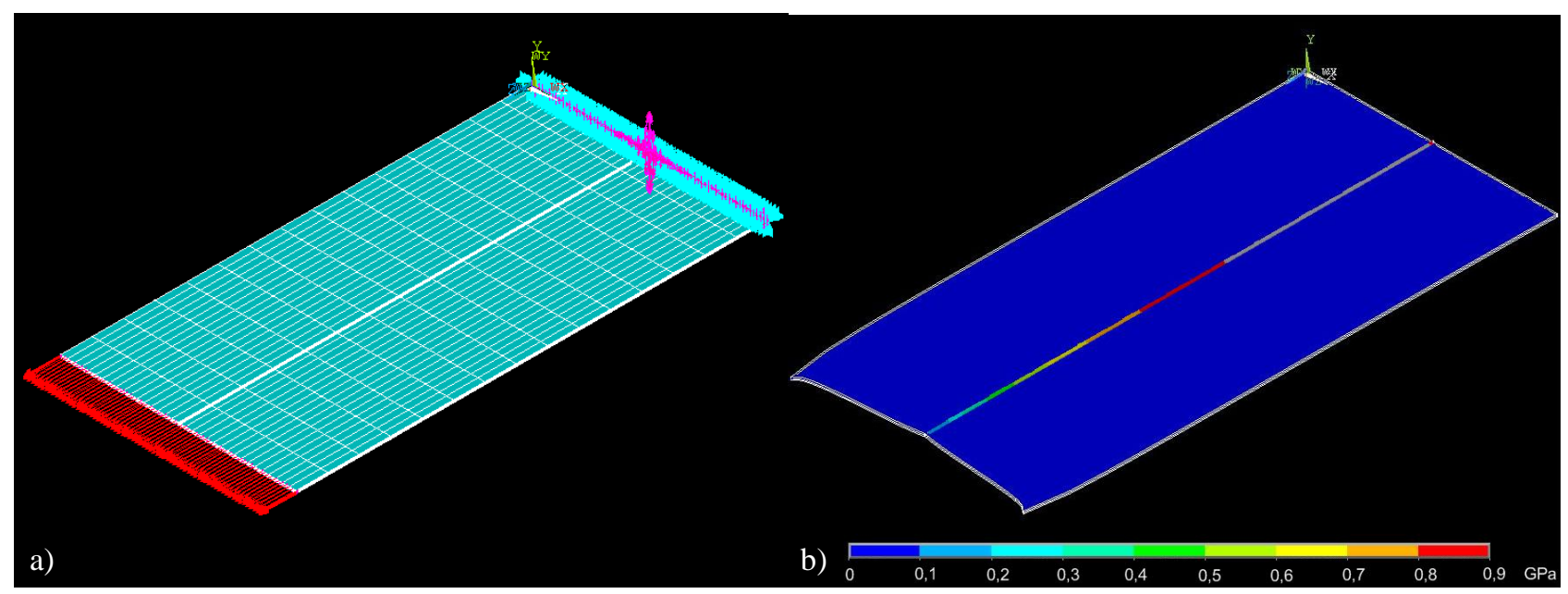

Fig. 2. Finite element model representation in the FEM software: a) model mesh with applied loads and constraints; b) nodal stress (von Mises yield criterion).

\section{Analysis}

The model was subjected to force loads applied at the 168 nodes that composes the limits of the polymer front face, as depicted by the red arrows in Fig. 2a. Besides the loads, it was also restraint at the back face of the polymer, by forcing a null displacement in the three directions. The force was applied as ramp function of $5 \times 10^{-3} \mathrm{~N}$ per second, at each node, over a 20 second period.

Figure $2 \mathrm{~b}$ shows the stress distribution at the middle section plane. The plane is at the half height of the model. It is interesting to see that, despite neither restraints nor direct forces were applied to the fiber, the polymeric foil deformation is sensed by the fiber, where the stress concentration was mainly in its middle section. The tensile effects in the fiber are more evident at the middle section, since the polymer drives the strain to that specific area. The sensor, according to these results, should be positioned in this region, where the effects were more significant.

From the available data, it is known that the fiber is capable of sustain a known yield strength of $0.7 \mathrm{GPa}$. To test fiber limits, during the analysis, it was subjected to higher stress. From Fig. 3a, the fiber passes above the yield strength when $8.5 \times 10^{-2} \mathrm{~N}$ is applied to the nodes. At this moment, the fiber presents a stretch of $600 \mu \mathrm{m}$. 


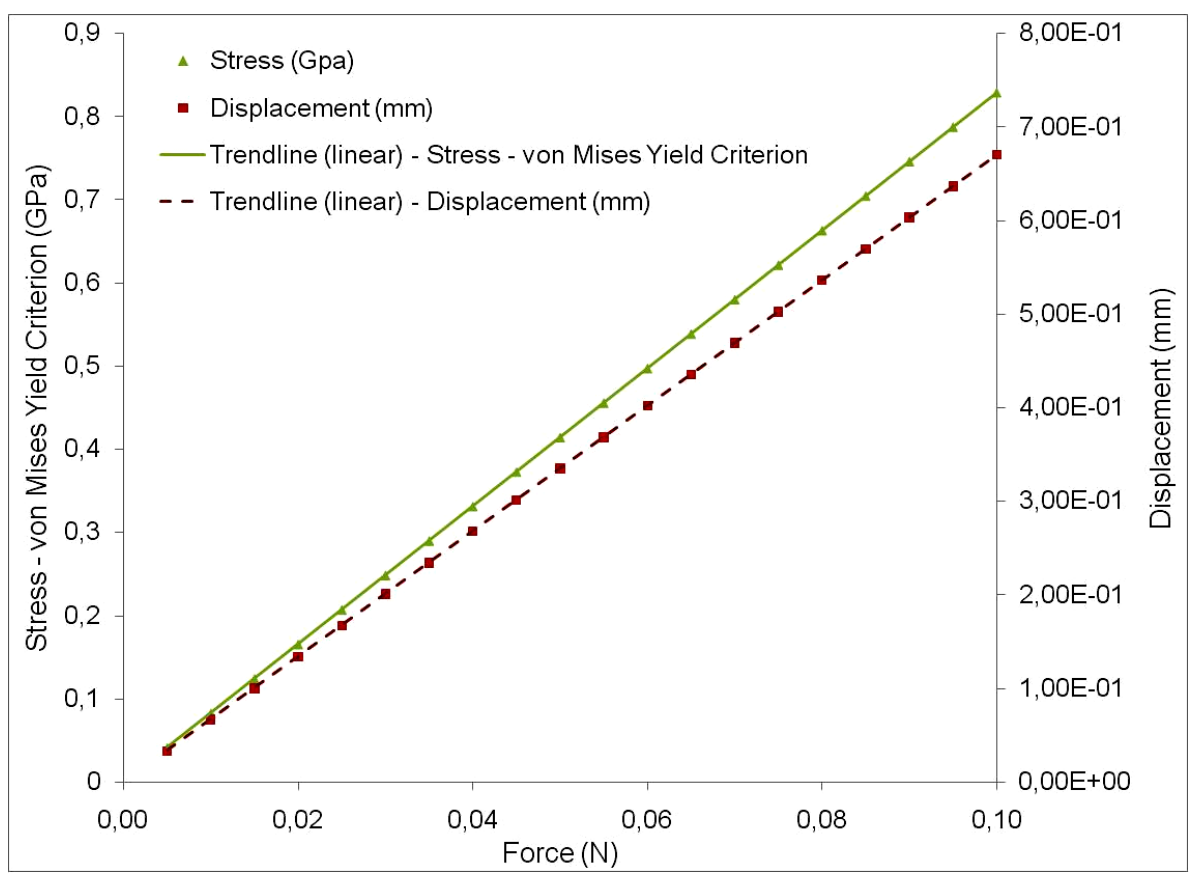

(a)

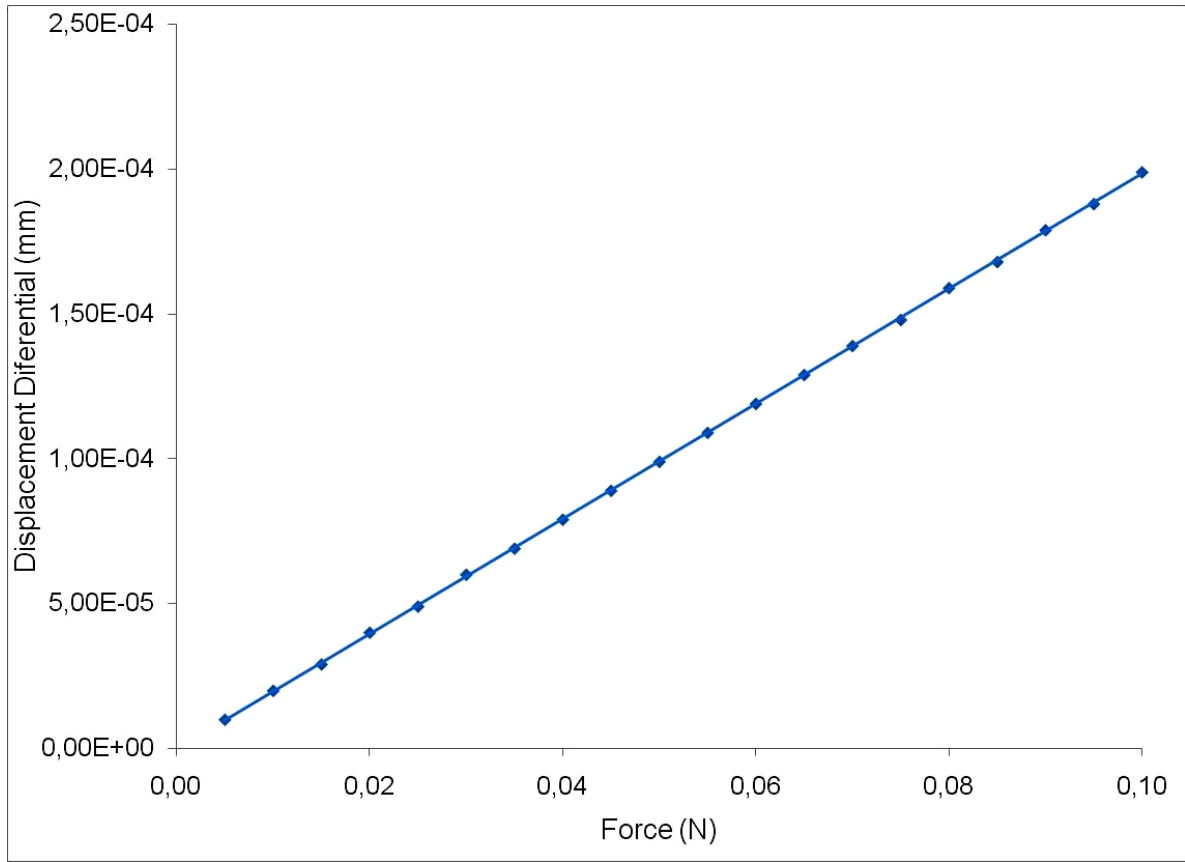

(b)

Fig. 3. (a) Stress and displacement variation over pressure application; (b) Displacement differential between a node at the polymer surface and a node at the interior of the fiber.

The polymeric foil does not present any significant stress due to its low Young's Modulus. This allows the PVC foil to be easily stretched. It is important that the polymer does not represent any obstacle for deformation transference. From the obtained and presented results, the fiber is fully sensitive to the deformation since it deforms along the polymer foil elongation. Besides, and yet more important, the difference between the polymer displacement and the fiber displacement is very small, ensuring that the foil deformation is completely transferred to the fiber sensor (Fig. 3b). 


\section{Sensing Device Prototype}

The optic fiber used in the prototype fabrication was a single mode Corning 28e, a standard fiber for communication applications, with acrylate coating. The Bragg structures were first printed in the fiber core using a communication wavelength range $(1520-1570 \mathrm{~nm})$. The fiber optic sensor was then packaged within a sandwiched polymeric foil (Fig. 4a). The fiber integration within the foil is a crucial step since it must be avoid any significant loss of sensor sensitivity due to the foil. The fabrication relies on an industrial process (Fig. 4b), where a first layer is applied in a structure that will work as substrate for the fabrication process. After the thickness homogenization, the layer is cured in the oven, to become a solid structure. The second layer suffers only a partial cure, in order to increase the viscosity and facilitate the insertion of the optic fiber. The third, and final layer, is placed over the fibers and is fully cured in the oven.

a)
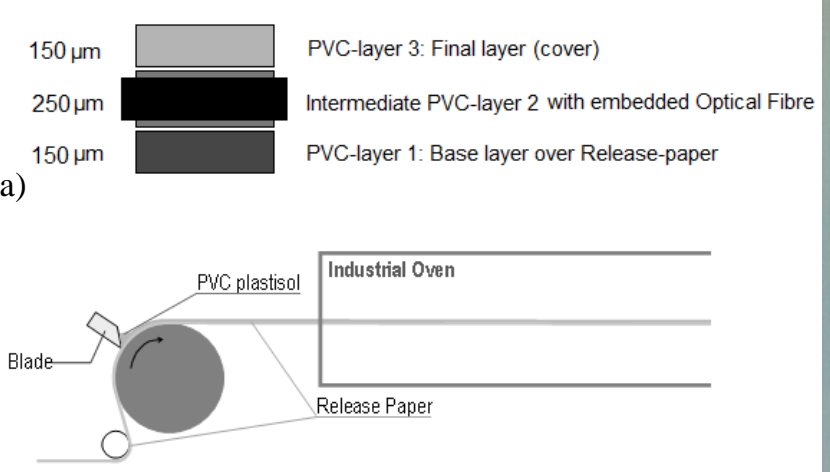

b)

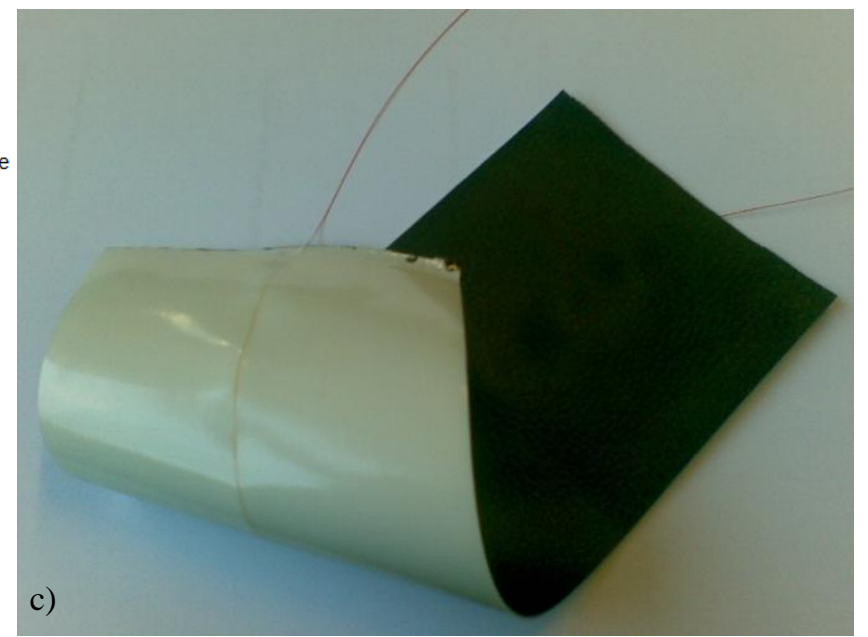

Fig. 4. a) Chosen approach and illustration; b) Industrial process schematic; c) Fabricated prototype.

\section{Measurements and Analysis}

Figure $4 \mathrm{~b}$ shows the functional sensing device prototype fabricated as previously described. The polymeric foil, 210x150 $\mathrm{mm}^{2}$ size, has a Bragg sensor embedded in it. By visual inspection no damage is detected, being a good indicator of the fabrication process success. By touch, the fiber is not felt, sustaining the thought of a good integration level. Figure 5a presents the reflected spectrum of the FBG sensor. The side lobes come from the grating fabrication process, resulting from radiation transmission function, and not from the integration process. The lobes can be later smoothed by apodization if necessary. When stretching the polymeric foil, the embedded FBG sensor follows the deformation and the reflected spectrum suffers a wavelength deviation. When the sample is released, the spectrum returns to the initial position.

Since one of the goals is to produce this type of foils with integrated sensors on industrial environments, the restraints of the industrial process had to be evaluated. Considering that optical sensors are, in general, sensitive to temperature, one of the analyzed restraints was the fabrication process temperature ranges. Several foils, with integrated optic fibers, were fabricated at different temperatures, from 200 to $240{ }^{\circ} \mathrm{C}$ with $20^{\circ} \mathrm{C}$ steps, and at different cures durations, from 60 to 180 seconds with 30 seconds steps. It was found that the PVC from the polymeric foil did not stand temperatures above $240{ }^{\circ} \mathrm{C}$ during 150 seconds. At the optic fiber level, all the Bragg sensors did support the temperature and the duration of the cure without presenting any sensitivity loss or damage. For the industrial process, these results does not present any restraints since, in general, the polymeric foils are fabricated at a temperature of $220^{\circ} \mathrm{C}$ for 60 seconds.

To better evaluate the performance of the produced model, the prototype was tested with an Instron ${ }^{\circledR} 4302$ testing machine at the same time that the optical signal was being measured by a BraggMETER ${ }^{\text {TM }}$ unit from FiberSensing company [8]. 

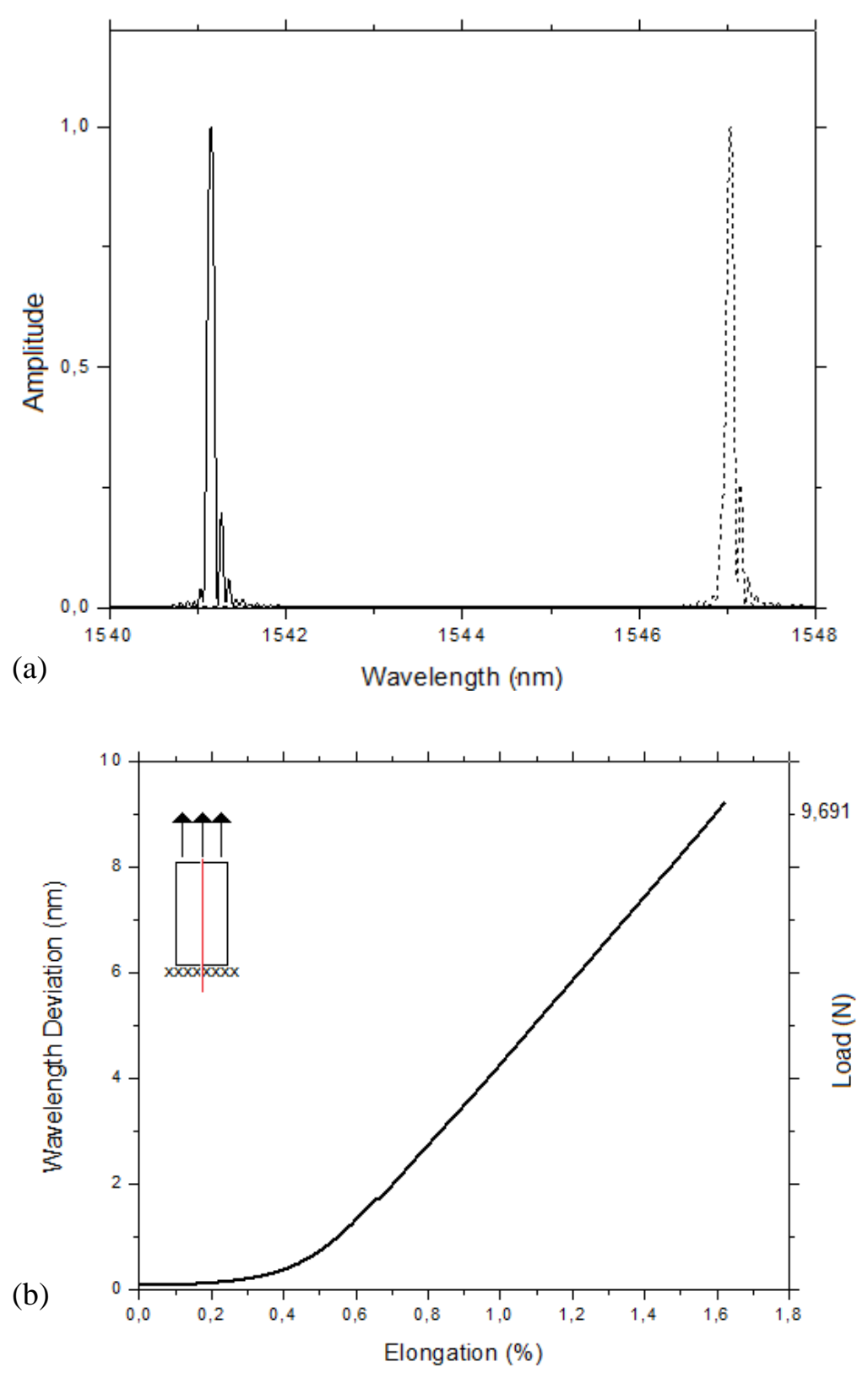

Fig. 1. (a) Reflected spectrum from the FBG sensor for two distinct tensile forces; (b) Bragg response to applied displacements.

The prototype was cropped to the $50 \times 100 \mathrm{~mm}$ size. During the test, the model was subject to a displacement at the rate of $16 \mu \mathrm{m} / \mathrm{s}$. As it is demonstrated over the graph (Fig. 5b), the wavelength deviation has a linear behavior from $0.5 \%$ onwards. Two reasons for the non-linear behavior below $0.4 \%$ can be pointed out. First, the testing machine does not have enough resolution for elongations lower than $400 \mu \mathrm{m}$, not providing precise elongation data, and in this case a test with a sample with double height may be used, since it duplicates the machine sensitivity. Second, the distance between the testing machine claws that hold the sample was inferior to the sample length. This leads to an initial stretch moment where the sample elongation is near $0 \%$ so, the wavelength deviation does not change much.

It was also verified that the prototype was able to sustain a stretching of $1.62 \%$ (strain), corresponding to $1.62 \mathrm{~mm}$ of displacement. In this situation, the fiber was subjected to a load of $9.691 \mathrm{~N}$.

A displacement of $1.62 \%$, with a wavelength deviation of $9.207 \mathrm{~nm}$, was measured and the sensitivity of the present prototype is $0.6 \mathrm{pm} / \mu \varepsilon$ (picometer per microstrain). In a different way, if we consider a 1 meter long steel beam that has been stretched $1 \mathrm{~mm}$, the wavelength deviation that 
would be measured is $0.6 \mathrm{~nm}$. The determined sensitivity value provides information about the integration quality.

\section{Conclusions}

In this paper, a finite element model and a fabricated prototype model were investigated and, although the results of each one may seem different, the overall model behavior was the same than the fabricated prototype. The finite element model provided relevant data about the stress distribution. This is crucial when dealing with the positioning of the Bragg sensor. It was showed that the difference between the deformation of the polymer and the fiber is very small. It is a good indicator for the loss of sensitivity that may exist. The fabricated prototype stretches a little more than the predicted by finite element model. This may be a result from the fact that the available material parameters do not match exactly the material properties of the fabricated device. For example, the PVC polymeric foil is a custom formulation from TMG Automotive ${ }^{\circledR}$. It was especially made for a better fiber integration level, which may result on different properties when compared to the commercial polymer. The fabricated prototype presented a sensitivity of $0.6 \mathrm{pm} / \mu$ strain, but the sensor network resolution will be always related to the monitoring system resolution. The structure showed good performance, not only the spectrum shape continued the same during the force application, but also showed good repeatability, since it returned to its initial position and there was not any reduction of the signal amplitude. At the integration level analysis, the results demonstrated the successful integration of fiber sensor within the polymeric foil.

Integration of sensors, as the Fiber Bragg Grating ones, in new substrates as polymeric foils or textiles laminated are proving to be a very fertile research area and new developments can be expected.

\section{Acknowledgements}

Alexandre Ferreira da Silva is supported by Portuguese Foundation for Science and Technology (SFRH/BD/39459/2007). This work is also sponsored by FCT/MIT-Pt/EDAM-SI/0025/2008.

\section{References}

[1] K.T.V. Grattan and T. Sun, "Fiber optic sensor technology: an overview," Sensors and Actuators a-Physical, vol. 82, pp. 40-61, May 152000

[2] O.S. Wolfbeis, "Fiber-optic chemical sensors and biosensors," Analytical Chemistry, vol. 78, pp. 3859-3873, Jun 152006

[3] R. Blue, et al., "Platform for enhanced detection efficiency in luminescence-based sensors," Electronics Letters, vol. 41, pp. 682-684, Jun 92005

[4] B. Glisic and D. Inaudi, Fibre Optic Methods for Structural Health Monitoring, 2007

[5] A.D. Kersey, et al., "Fiber grating sensors," Lightwave Technology, Journal of, vol. 15, pp. 1442-1463, 1997

[6] N.H. Rizvi and M.C. Gower, "Production of Bragg gratings in optical fibres by holographic and mask projection methods," in Optical Fibre Gratings and Their Applications, IEEE Colloquium on, 1995 , pp. $3 / 1-3 / 6$

[7] E.M. Petrie. (2005, PVC Plastisol Adhesives. Adhesives \& Sealants

[8] A. Rodolfo Jr., et al., Tecnologia do PVC, 2 ed.: Braskem S.A., 2006 


\section{Advanced Materials Forum V}

doi:10.4028/www.scientific.net/MSF.636-637

Fiber Bragg Grating Sensors Integrated in Polymeric Foils

doi:10.4028/www.scientific.net/MSF.636-637.1548

\section{References}

[1] K.T.V. Grattan and T. Sun, "Fiber optic sensor technology: an overview," Sensors and Actuators a-Physical, vol. 82, pp. 40-61, May 152000

doi:10.1016/S0924-4247(99)00368-4

[2] O.S. Wolfbeis, "Fiber-optic chemical sensors and biosensors," Analytical Chemistry, vol. 78, pp. 3859-3873, Jun 152006

doi:10.1021/ac060490z

PMid:16771528

[3] R. Blue, et al., "Platform for enhanced detection efficiency in luminescence-based sensors," Electronics Letters, vol. 41, pp. 682-684, Jun 92005 doi:10.1049/el:20050744

[4] B. Glisic and D. Inaudi, Fibre Optic Methods for Structural Health Monitoring, 2007

[5] A.D. Kersey, et al., "Fiber grating sensors," Lightwave Technology, Journal of, vol. 15, pp. 1442-1463, 1997

[6] N.H. Rizvi and M.C. Gower, "Production of Bragg gratings in optical fibres by holographic and mask projection methods," in Optical Fibre Gratings and Their Applications, IEEE Colloquium on, 1995, pp. 3/1-3/6

[7] E.M. Petrie. (2005, PVC Plastisol Adhesives. Adhesives \& Sealants

[8] A. Rodolfo Jr., et al., Tecnologia do PVC, 2 ed.: Braskem S.A., 2006 